\title{
Spatial characterization of raincell horizontal profiles from C-band radar measurements at mid-latitude
}

\author{
M. Montopoli ${ }^{1}$, F. S. Marzano ${ }^{1,2}$, G. Vulpiani ${ }^{1}$, A. Fornasiero ${ }^{3,4}$, P. P. Alberoni ${ }^{3}$, L. Ferraris ${ }^{4}$, and N. Rebora ${ }^{4}$ \\ ${ }^{1}$ Dipartimento di Ingegneria Elettrica - Centro di Eccellenza CETEMPS, Università dell'Aquila, L'Aquila, Italy \\ ${ }^{2}$ Dipartimento di Ingegneria Elettronica - Università "La Sapienza" di Roma, Rome, Italy \\ ${ }^{3}$ Servizio Idro-Meteorologico ARPA Emilia Romagna, Bolgna, Italy \\ ${ }^{4}$ CIMA, Università di Genova e della Basilicata, Savona, Italy
}

Received: 11 November 2005 - Revised: 2 February 2006 - Accepted: 14 February 2006 - Published: 18 April 2006

\begin{abstract}
A spatial characterization of mid-latitude mesoscale rain fields from $\mathrm{C}$-band radar measurements is performed by means of a systematic analysis and modelling of convective raincell shapes. To this aim a large rainfall dataset, derived from an operational C-band dualpolarized radar, has been continuously collected from 1996 to 1999 . The radar-derived rain fields consist of 1558 grids of $256 \times 256 \mathrm{~km}^{2}$ with a spatial resolution of $1 \mathrm{~km}$. A new accurate and adaptive algorithm for raincell identification is introduced and thoroughly discussed. From this analysis, a quality-controlled set of 2601 raincells, together with the radial rain intensities (or raincell horizontal profiles), is extracted. Three one-dimensional analytical models of rainfall horizontal profile are reviewed and tested by best fitting their parameters against estimated raincell data. The statistical results of this intercomparison are quantitatively analyzed and discussed in terms of mean rainfall horizontal profiles and root mean square errors.
\end{abstract}

\section{Introduction}

Spatial characterization of rainfall patterns has become important in various fields, such as meteorology, hydrology and radio propagation (Austin and Houze, 1972; Bell, 1987; Crane 1990; Marzano et al., 2001). As an example, in hydrology the interest to model rain fields is mostly due to the need of initializing run-off models and to devise downscaling methods in order to properly deal with sub-basin scales for flood forecast (Veneziano et al., 1996; Willems, 2001). Furthermore, spatial rain cell description is very important for flood risk assessment of operational forecast systems, rainfall classification schemes and for rain temporal characterization within tracking procedures (Rigo land Llasat, 2004). Apart

Correspondence to: $\mathrm{M}$. Montopoli

(mmontop@ing.univaq.it) from the specific purpose, in order to model rain fields in a realistic way it is necessary to resort to highly spatially resolved rainfall data, possibly derived from the integration of weather radar, satellite sensor and raingauge network measurements (Meija and Rodriguez-Iturbe, 1974; Wheater et al., 2000; Ferraris et al., 2003; Marzano et al., 2001, 2002).

Weather microwave radars are powerful tools for rain field monitoring due to their characteristics of wide areal coverage, high sensitivity to hydrometeor electromagnetic backscattering and fairly high spatial resolution, especially at short to medium ranges (i.e. up to $125 \mathrm{~km}$ from the radar site). Indeed, the accuracy of surface rain fields estimation can be questionable and should be carefully considered under some circumstances, as in the case of beam blockage, enhanced ground-clutter, anomalous propagation, bright band contamination, strong path attenuation, second-trip echo ambiguity and long-range error bias (e.g., Alberoni et al., 2001; Marzano et al., 2004). Moreover, the relation between radar backscattered power and rain intensity may depend on precipitation microphysical processes (e.g., Sauvageot, 1992). Nevertheless, the radar-derived estimates can be considered nowadays the major source for a robust spatial-temporal characterization of rainfall features.

The automatic identification of a raincell from a radar image is not a simple task. Rainfall patterns are quite complex and, indeed, a convective raincell can exist either alone at various stages (e.g., supercell) or within an organized cluster or embedded in a stratiform region (Rigo and Llsat, 2004). An identification of rainfall pattern can be performed by using either a bi- (2-D) or tri-dimensional (3-D) radar dataset. In this work we have limited our attention to radar-based 2-D techniques. The latter are usually designed so that a rainfall field is detected by means of a rainfall constant threshold (Steiner et al., 1995). This approach is significantly prone to the inclusion of sub-raincells or secondary maxima within a single raincell. Several efforts have been also devoted to 
model the average horizontal profile of convective raincells. Mid-latitude radar observations (Fèral et al., 2003) showed that the rain rate horizontal distribution near the raincell peak (its maximum) does not sharply decay as an exponential function, but rather as a Gaussian one or as a combination of these two functions. On the other hand, others works (Von Hardenberg et al., 2003) showed that, on average and in a tropical regime, the rainfall intensity decreases exponentially from the raincell peak. These different results are probably due to different data processing and to the climatology of the measured raincells so that they cannot be generalized in a straightforward manner.

The aim of the present work is to characterize the average one-dimensional (1-D) horizontal structure of raincells derived from weather radar images collected during four years of operational activity, from 1996 to 1999, at mid-latitude. With the previous issues in mind, the focus is twofold: i) to set up a new accurate and objective procedure for convective raincell identification; ii) to select the best analytical model for describing the average 1-D raincell horizontal profile using the available radar dataset. Section 2 is devoted to illustrate the radar characteristics, the rainfield retrieval procedure and the new raincell recognition algorithm. In Sect. 3 the 1-D analysis of the average raincell shape is carried out on all single-peaked raincells, previously identified, and the three different shape models are introduced. The conclusions, in Sect. 4, summarize the differences between the three raincell models implemented.

\section{Raincell automatic recognition methodology}

In this section, we will describe a methodology and a procedure to recognize convective raincells in an automatic way. Raincell patterns are derived from plan position indicator (PPI) scans at the lowest elevation angle of a meteorological C-band radar. Special attention will be devoted to isolate single raincells belonging to a cluster of raincells.

\subsection{Radar data pre-processing}

Rainfall data were provided by the C-band operational Doppler of S. Pietro Capo fiume (Bologna, Italy), located along the Po-river valley in northem Italy (Alberoni et al., 2001). This dual-polarization radar is placed on a tower with a Cassegrain parabolic antenna (without radome cover), providing a half-power beam-width of $1.0^{\circ}$ and a directivity of about $45-\mathrm{dB}$. The klystron peak-power is $250 \mathrm{~kW}$ at $5.6 \mathrm{GHz}$ with an alternating horizontal-vertical polarization transmission and a dual pulse repetition frequency (PRF) system for unfolding capability. Pulse widths of $0.5 \mathrm{~ms}$ (i.e., short pulse with a re-sampled bin resolution of $250 \mathrm{~m}$ ) and $1.5 \mathrm{~ms}$ (i.e., long pulse with a re-sampled bin resolution of $1500 \mathrm{~m}$ ). The receiver sensitivity is equal to $113 \mathrm{dBm}$. The typically used maximum range is $250 \mathrm{~km}$ (with long pulse) and $125 \mathrm{~km}$ (with short pulse) for the intensity and velocity mode, respectively.
Radar data are acquired with a prescribed scanning strategy during operational activities, consisting of 15 elevations with an angular spacing of $1^{\circ}$. Radial spatial resolution is set to $250 \mathrm{~m}$ for short ranges (i.e., $125 \mathrm{~km}$ ) and to $1 \mathrm{~km}$ for long range (i.e., $250 \mathrm{~km}$ ) scans, the latter being carried out only twice per hour. Time sampling of radar volume data is such that there are 4 acquisitions per hour (i.e., every $15 \mathrm{~min}$ ) being the dual-polarized one performed only twice per hour. Procedures to correct for gas absorption, to remove groundclutter echoes and to identify anomalous propagation conditions are routinely applied. Side-lobe effects at very short ranges (less than $20 \mathrm{~km}$ ) for low elevations are avoided by choosing higher elevations not affected by this effect (Alberoni et al., 2001).

Four years of radar data, acquired from January 1996 to December 1999 in an operational mode, have been considered in the present study. We have used only radar reflectivity at horizontal polarization, here indicated by $Z_{h h}(r, \theta, \varphi)$ or simply $Z(r, \theta, \varphi)$, where $r$ is the range, $\theta$ the elevation angle $\theta$ and $\varphi$ the azimuth angle $\varphi$. The radial resolution and the maximum range have been set, respectively, to $250 \mathrm{~m}$ and $125 \mathrm{~km}$. Due to orography blockage in the southwestern sector of observation, at each range-azimuth bin location we have extracted the value of $Z(r, \theta, \varphi)$ relative to the lowest available elevation $\theta_{m}$ using a radar visibility map. The resulting polar map of measured lowest-bin $Z\left(r, \theta_{m}, \varphi\right)$ has been then projected on a regular $256 \times 256-\mathrm{km}^{2}$ Cartesian grid with 1-km horizontal resolution and $(i, j)$ the indexes along $x$ and $y$, respectively. In case more than one $Z\left(r, \theta_{m}, \varphi\right)$ value belongs to the same Cartesian pixel $(i, j)$, an average $Z\left(r, \theta_{m}, \varphi\right)$ value has been attributed to the pixel itself applying the nearest-neighbourhood algorithm (Pratt, 1991). The output of this procedure has been the Cartesian map of the reflectivity factor $Z(i, j)$ at each available time step. $\Delta r$ For an easy representation and interpretation, $Z(i, j)$ has been converted to surface rainrate $R(i, j)$, using a standard power law Z-R relation: $R(i, j)=a \cdot[Z(i, j)]^{b}$. The latter has been computed for each pixel $(i, j)$ and with the coefficients $a$ and $b$ equal to those of Marshall-Palmer (i.e., $a=200, b=1.6$ with $R$ in $\mathrm{mm} / \mathrm{h}$ and $Z$ in $\mathrm{mm}^{6} \mathrm{~m}^{-3}$ ). As a result, the measured dataset has been converted into a temporal series of 1558 rainrate maps $R$ (or frames) of $256 \times 256$ pixels.

\subsection{Raincell identification algorithm}

In this section a new raincell-identification algorithm is illustrated in detail. By means of this procedure, we have automatically extracted 2601 raincells from the 1558 measured rainfall Cartesian maps previously introduced. This raincell extraction is essential before proceeding to any quantitative evaluation of the shape of each raincell. The basic philosophy of the proposed raincell recognition algorithm is to introduce a variable lower threshold on each rainrate map in order to delimitate single raincells which are, potentially, the convective cores. 
The automatic identification algorithm is composed by the following 8 steps repeated for all 1558 frames.

1. Finding all locations of the local maxima of the rainfall field such that, for each pixel $\left(i_{M}, j_{M}\right)$, the rainrate local peak $R\left(i_{M}, j_{M}\right)$ is larger than both its 8-connected neighbor-pixel values and the threshold of $10 \mathrm{~mm} / \mathrm{h}$.

2. Sorting the local maxima $R\left(i_{M}, j_{M}\right)$ in a decreasing order so that it is possible to privilege the highest values of raincell peaks.

3. Selecting a pixel box of size $N \times N$ of the rain field $R$ in order to define the sub-field $R_{b}$ within the box. At each algorithm iteration, the center pixel of $R_{b}(i, j)$ is positioned on the current maximum location $\left(i_{M}, j_{M}\right)$. Note that past analyses of radar data showed that raincells larger than about $20 \mathrm{~km}$ in equivalent diameter are not numerous (Mesnard and Sauvageot, 2003, Féral et al., 2000). Therefore, we have chosen the box size $N$ equals to 25 to include in $R_{b}$ the major portion of raincells in order to capture its main spatial characteristics.

4. Computing the 2-D normalized autocorrelation function $\left(C_{R b}\right)$ of $R_{b}$ in order to extract an adaptive lower threshold value $\left(R_{t}\right.$ low $)$ for the raincell delimitation. This step is crucial as, in this way, the raincell area is strictly linked to the local spatial texture of raincell itself. If we utilize an upper-left-corner justified definition for the $R_{b}$ matrix, we can define the pixel $-\operatorname{lag} m$ (or $n$ ) as the discrete distance between the $R_{b}$ upper left corner pixel and the lower left (or upper right) corner pixel of its shifted version $R_{b}(i-m+N, j-n+N)$ (Pratt, 1991). With these definitions in mind, the 2-D normalized autocorrelation function $C_{R b}(m, n)$, for a given lag $m$ and $n$, of the box-sized rain field $R_{b}(i, j)$ is computed $I$ as follows:

$C_{R_{b}}(m, n)=\frac{\sum_{i} \sum_{j} R_{b}(i, j) \cdot R_{b}\left(i-m+N, j-n_{N}\right)}{\sum_{i} \sum_{j} R_{b}^{2}(i, j)}$

where the indexes $i$ and $j$ vary in the ranges $\operatorname{Max}[1, m-N+1] \leq i \leq \operatorname{Min}[N, m]$ and $\operatorname{Max}[1, n-N+1] \leq j \leq \operatorname{Min}[N, n] \quad$ with $\operatorname{Max}$ and Min denoting the maximum and the minimum of the arguments, respectively. We emphasize that the indexes $m$ and $n$ indicate the pixel-lags and vary in the range $[1,2 N-1]$ because $R_{b}(i-m+N, j-n+N)$ moves in space over $R_{b}(i, j)$. Accordingly, $C_{R b}(m, n)$ is an element of a matrix of size $(2 N-1) \times(2 N-1)$ which describes the spatial features of $R_{b}(i, j)$ of the current frame. Note that, for $m=n=N, C_{R b}(N, N)$ is equal to unity by definition.

5. Averaging radial sections of the 1-D autocorrelation $C_{R b}$ in order to derive the correlation length of the boxsized rain field $R_{b}$, as described in the following step.
If $l$ is the index along the $k$-th radial section $s_{k}$ of $C_{r b}$ for each raincell local maximum, taking four sections $C_{R b}\left(l ; s_{k}\right)$ of $C_{R b}$ along x-axis, y-axis, positive bisector and negative bisector directions, the average onedimensional radial autocorrelation $C_{R r}(l)$ is defined as:

$C_{R_{r}}(l)=\frac{1}{4} \sum_{k=1}^{4} C_{R_{b}}\left(l ; s_{k}\right)=\frac{1}{4} \sum_{k=1}^{4} C_{R_{b}}\left(m, n ; s_{k}\right)$

where $C_{R b}\left(m, n ; s_{k}\right)$ is $C_{R b}(m, n)$ along the $k$-th section and $l$ varies in the range $[1,2 N-1]$ along each section. The $C_{R r}(l)$ maximum is reached when $l=N$ and it is equal to unity.

6. Computing the average correlation length $L_{c}$ of $C_{R r}(l)$ defined as the discrete distance from the $C_{R r}$ maximum location (equal to $l=N$ ) such that $C_{R r}(l)$ is reduced of about $36 \%$. By defining an additional square sub-box centered on the current maximum $\left(i_{M}, j_{M}\right)$ of size $\left(2 L_{c}-1\right) \times\left(2 L_{c}-1\right)$, a lower threshold $R_{t}$ low can be finally computed as an average rain rate of $R_{b}(i, j)$ within this sub-box.

7. Identifying the raincell field $R_{c}$, where the subscript $c$ stands for raincell, starting from the current maxima $\left(i_{M}, j_{M}\right)$ and considering the 8-connected values of $R_{c}(i, j)$ larger than $R_{t \text { low. }}$. The threshold $R_{t \text { low }}$ is automatically set to $5 \mathrm{~mm} / \mathrm{h}$ if this value is overtaken negatively.

8. The previous steps from 3 to 7 are repeated for all local maxima identified in the first step. Note that the lower threshold $R_{t \text { low }}$ is not the only threshold that needs to be fixed within this procedure. The pixels belonging to a generic raincell must be constrained to further limitations such as:

i) all the pixel values of a given raincell cannot overcome the raincell maximum;

ii) all the pixel values of a given raincell cannot be larger than the lower threshold of the nearest cells already identified.

Furthermore, we have to remark that sizes of $R_{b}$ larger than 25 pixels have been also explored, but this leads to smaller values of $R_{t}$ low. If $R_{t}$ low is too small, it becomes almost impossible to delineate a single raincell. For this reason, we have chosen, as a compromise, the $R_{b}$ size equal to $25 \mathrm{~km}$.

An example of the identification algorithm results is shown in Fig. 1. This figure displays different representations of an extracted raincell, underlining its principal axes and orientation as defined in Sect. 3. Notice that the threshold $R_{t}$ low has a spatial-correlation dependence and this feature tends to ensure a fairly accurate single raincell identification even when belonging to a cluster of raincells. As a matter of fact, it is worth mentioning that these raincells may have multiple 

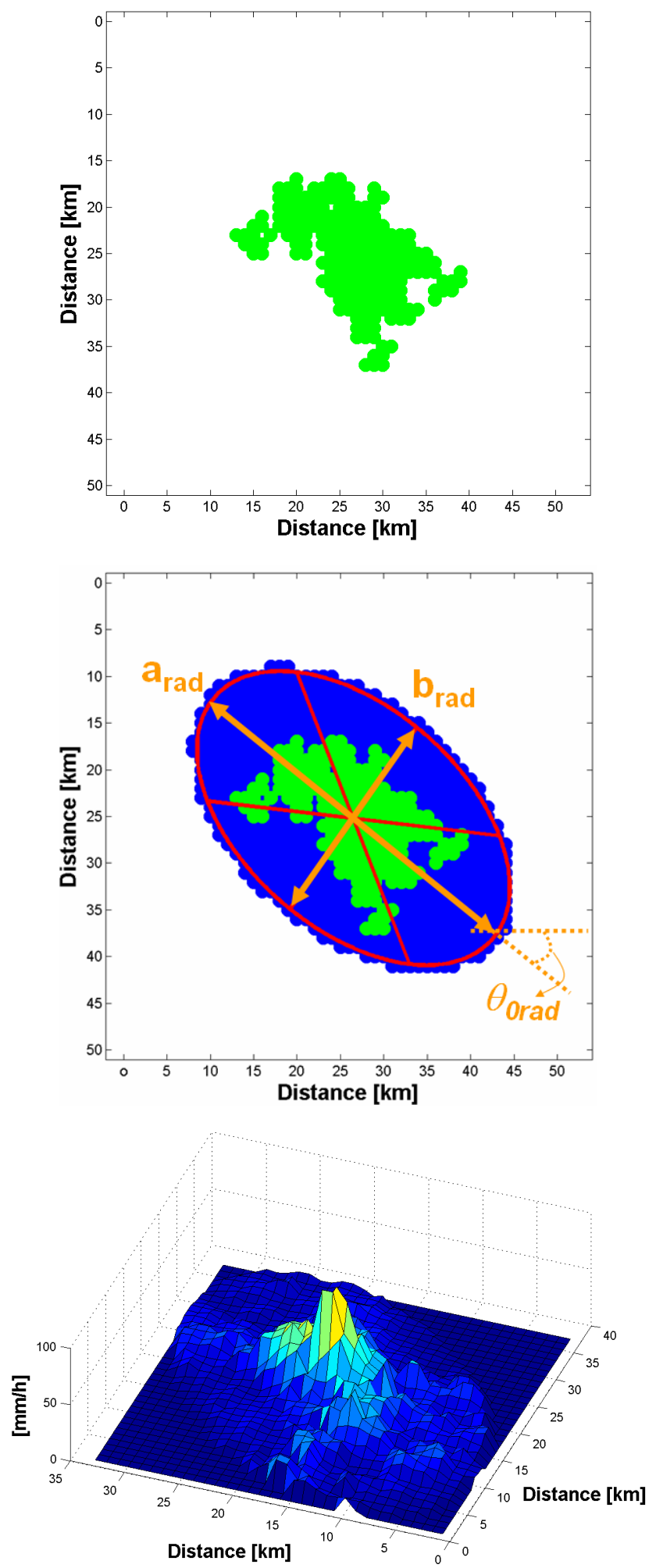

Fig. 1. An example of raincell identification. On the top panel, the pixels belonging to the generic raincell $(R(i, j)$ over threshold $\left.R_{t \text { low }}\right)$ are shown. On the middle panel, the principal axes $a_{\text {rad }}$, $b_{\text {rad }}$ and the orientation $\theta_{0 \text { rad }}$ are superimposed to the raincell and its surrounding area. On the bottom panel, a pattern example of real raincell extracted is shown. rainrate peaks, as clarified later on. From the previous analysis applied to the whole available radar dataset, a set of 2601 raincells has been finally extracted.

\section{Modelling raincell horizontal profiles}

A common choice to model raincell horizontal profiles (or raincell shapes) is to resort to an elliptical perimeter centered in the raincell peak. From available C-band radar data, we have derived an average horizontal profile of rainrate and tried to best fit these experimental shapes with three analytical rainrate profile models, as explained later on.

\subsection{Horizontal profile estimation from radar data}

In the following text the subscript "rad" will stand for "radarderived" estimated quantity in order to distinguish it from the corresponding modeled one. For each identified raincell, the principal axes and orientation have been calculated as follows:

$m_{p q}=\sum_{i} \sum_{j}\left(i-i_{C}\right)^{p}\left(j-j_{C}\right)^{q} R_{c r a d}(i, j)$

$a_{\mathrm{rad}}=\left\{\frac{\left[m_{20}+m_{02}\right]+\sqrt{\left(m_{20}-m_{02}\right)^{2}+4 \cdot m_{11}}}{2 m_{00}}\right\}^{1 / 2}$

$b_{\mathrm{rad}}=\left\{\frac{\left[m_{20}+m_{02}\right]-\sqrt{\left(m_{20}-m_{02}\right)^{2}+4 \cdot m_{11}}}{2 m_{00}}\right\}^{1 / 2}$

$\theta_{0 \mathrm{rad}}=\frac{\pi}{2}-\operatorname{tg}^{-1}\left(-\frac{m_{11}}{m_{22}-a}\right) \quad$ with $\quad \theta_{0 \mathrm{rad}} \in[0, \pi)$

where $\left(i_{C}, j_{C}\right)$ is the centroid pixel of the generic raincell (Von Hardenberg et al., 2003), $R_{c \text { rad }}(i, j)$ are the rain intensities within the raincell (see Sect. 2.2), $m_{p q}$ is the centered momentum with $p$ and $q$ momentum order, $a_{\mathrm{rad}}$ and $b_{\mathrm{rad}}$ are the principal and secondary axis lengths, respectively, and $\theta_{0 \text { rad }}$ is the orientation angle of the raincell defined as in Fig. 1.

Figure 2 shows the distribution of estimated $\theta_{0 \text { rad }}$ orientation and ellipticity $e_{\text {rad }}$, defined as the ratio $e_{\mathrm{rad}}=b_{\mathrm{rad}} / a_{\mathrm{rad}}$. Our analysis confirms the results obtained by Von Hardenberg et al. (2003) and Féral et al. (2000), showing a uniform distribution of raincell orientations and a mean ellipticity of about 0.5 (0.48 in this analysis).

Besides the principal axes, we have also extracted the two sections rotated at $45^{\circ}$ with respect to the principal axes themselves. Once these sections defined, the rainrate horizontal profiles along them have been extracted and then averaged to obtain an azimuthally-mean rain-rate horizontal profile $R_{c \text { rad }}(l)$. Here the coordinate $l$ is, of course, the discretized radial distance from the center of the single-peak raincell. 

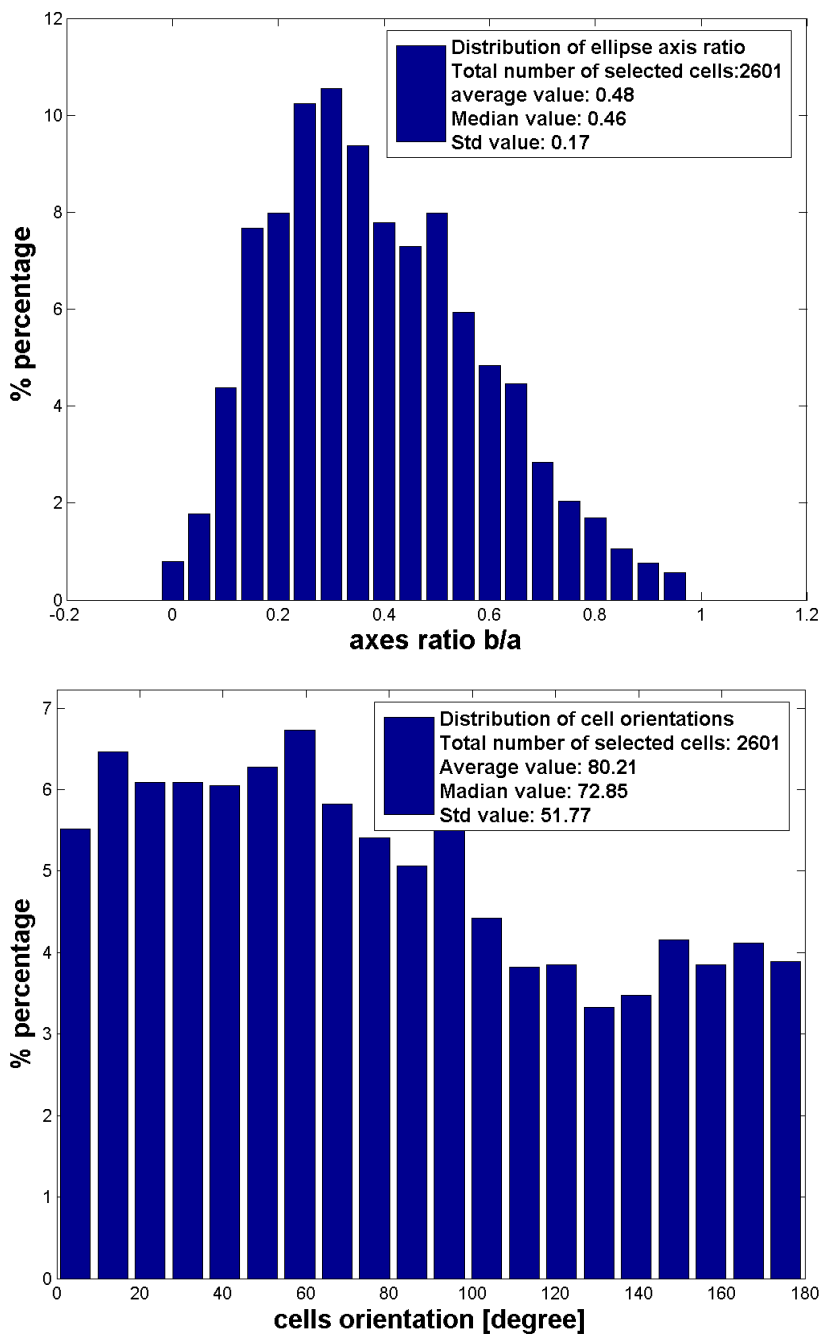

Fig. 2. Histogram of the 2601 raincells identified by means of the proposed method. On the top panel, the distribution of the ellipticity ratio $e_{\mathrm{rad}}=b_{\mathrm{rad}} / a_{\mathrm{rad}}$ is shown (with a mean value equals to 0.48 ). On the bottom panel, the raincell orientation distribution $\theta_{0 \mathrm{rad}}$ is shown (with an apparent uniform distribution).

To identify only the decreasing average monotone shapes, a pre-analysis on the existence of multi-peak structures has been also performed. The approach has been based on the computation of the spatial derivative of each single raincell shape $R_{c \text { rad }}(l)$ in order to identify local secondary maxima. Only the $28 \%$ of total raincells detected have shown a single peak trend and our study has been focused on this subset of measurements. Single-peak raincell fields, obtained from radar data processing described above, have been indicated by $R_{c s \text { rad }}(l)$. A total number of radar-derived 2992 horizontal profiles (corresponding to 748 raincells) has been processed and the overall average horizontal rainrate profile $<R_{c s \text { rad }}(l)>$ is shown in Fig. 3 by dotted line where the angle brackets stand for ensemble averaging of all available profiles.
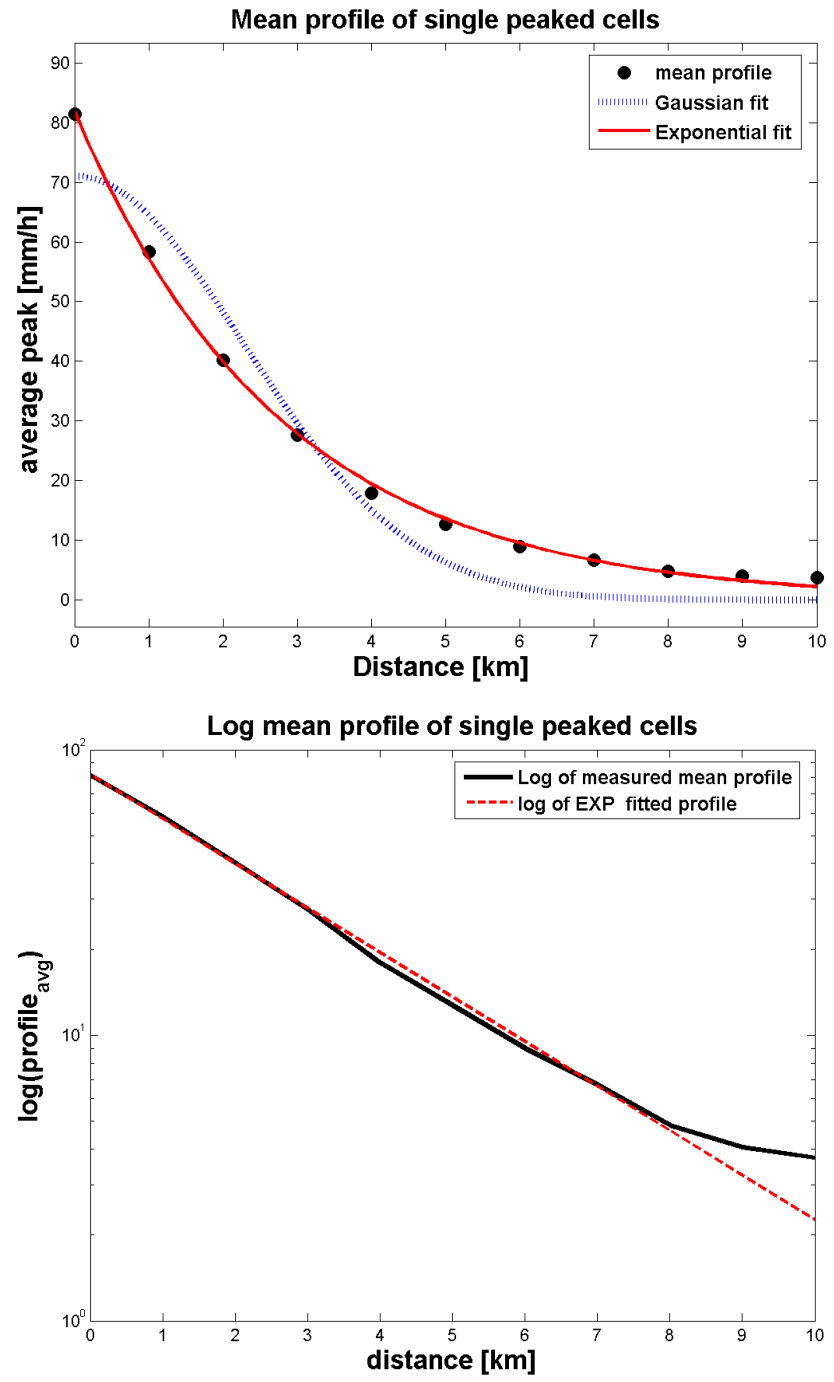

Fig. 3. On the top panel the ensemble average of the 2992 singlepek raincell shapes $\left\langle R_{c s \text { rad }}(r)>\right.$ (black dots) is shown. The red solid line is the best exponential fitting, whereas the dotted blue line is the average Gaussian best fitting profile $<R_{c s \mathrm{GAU}}(r)>$. On the bottom panel, the plot of the $\log <R_{c s} \operatorname{EXP}(r)>$ (dotted line), and the logarithm of mean shape $\log <R_{c s \text { rad }}(r)>$ (solid line) is shown. Both graphics indicate the exponential dependence of the shapes over at least up to $8 \mathrm{~km}$ distance.

\subsection{Analytical models of rainrate horizontal profiles}

As mentioned, three analytical one-dimensional models of rainrate horizontal profile $R_{c S}(l)$, derived from available literature, have been tested: the exponential (EXP), described by Capsoni et al. (1987), the Gaussian (GAU), described by Von Hardenberg et al. (2003) and the hybrid (HYB), described by Feral et al. (2003). The HYB is a combination of the GAU and EXP models with a rainrate threshold separating the two behaviors. The details of these one-dimensional models are given in the Appendix for completeness Here it suffices to underline that: i) the EXP and GAU model are 2-parameter models; ii) the HYB is a 4-parameter model. It 


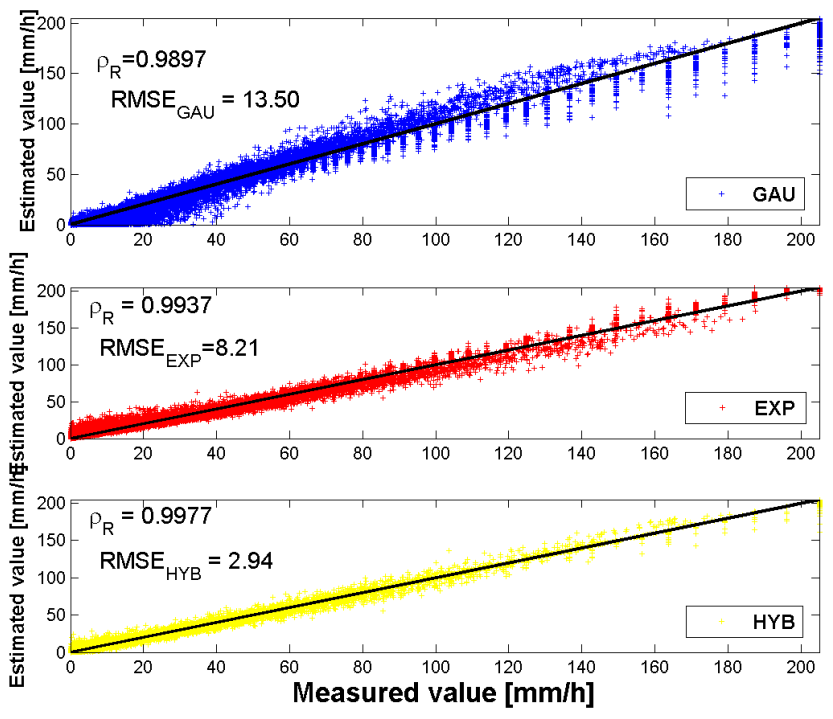

Fig. 4. Scatterplots between observed and fitted points of all 2992 single-peak shapes. From upper to lower panel it is shown the Gaussian GAU profile model (ARMSE $=13.5 \mathrm{~mm} / \mathrm{h}, \rho_{R}=0.9897$ ), the exponential GAU profile model $\left(\mathrm{RMSE}=8.21 \mathrm{~mm} / \mathrm{h}, \rho_{R}=0.9937\right)$, and the hybrid HYB profile model (ARMSE $=2.94 \mathrm{~mm} / \mathrm{h}$, $\left.\rho_{R}=0.9977\right)$.

should be mentioned that the all models are, indeed, a 2-D rainrate pattern models, but here we share the objectives of Von Hardenberg et al. (2003) limiting our attention to model the average 1-D horizontal rainrate profile.

In order to evaluate the goodness of each raincell 1-D model, the parameters of the latter have been retrieved by a best-fitting procedure. The result of the fitting process is an estimate of the unknown coefficients of the three rainrate models. To obtain the coefficient estimates of the n-th raincell horizontal profile $R_{c s}^{(n)}(l)$, we have minimized the summed square of residuals represented by the following metrics $d$ :

$d=\sum_{l=1}^{N_{r}}\left(R_{c s \mathrm{rad}}^{(n)}(l)-R_{c s \mathrm{MOD}}^{(n)}(l)\right)^{2}$

where $N_{r}$ is the number of profile radial points of the n-th raincell $R_{c s}^{(n)}$. From Eq. (7) it is clear that a residual is defined as the difference, at the discrete distance $l$ from the raincell peak, between the $\mathrm{n}$-th radar-observed horizontal profile value $R_{c s r a d}^{(n)}(l)$ and the $\mathrm{n}$-th modeled horizontal profile value $R_{c s \mathrm{MOD}}^{(n)}(l)$ (where MOD stands for EXP, GAU, and HYB).

It is worth recalling that the number of free parameters of 1-D raincell model to be retrieved from radar measurements are different (i.e., 2 for EXP and GAU and 4 for HYB). Moreover, for the HYB model there is an increase of the number of parameters due to its higher complexity. In fact, $R_{c s \mathrm{HYB}}^{(n)}(l)$ is the combination of two functions and the parameter retrievals involve two minimization processes under the constraint expresses from the continuity condition at the interface between the 2 different shapes of the hybrid model (see Eq.(A4) reported in the Appendix).
The left panel of Fig. 3 provides the average estimated horizontal profile $<R_{c s \text { rad }}(l)>$ superimposed to Exponential $<R_{c s \operatorname{EXP}}(l)>$ and Gaussian $<R_{c s \mathrm{GAU}}(l)>$ best-fitting model curves. On the right panel of the same figure it is plotted the logarithm of the average shape compared with the pure exponential trend which results to be a straight line in this plot. From this figure we can appreciate the good data fit of the EXP model with respect to the GAU one, at least up to a 8$\mathrm{km}$ distance. On the other hand, the GAU model tends to underestimate the average estimated rainrate maximum.

Figure 4, shows a scatterplot of the horizontal profile due to the three models, that is $R_{c s \operatorname{EXP}}(l), R_{c s \mathrm{GAU}}(l)$ and $R_{c s \mathrm{HYB}}(l), R_{\exp }(r) R_{\text {gauss }}(r) R_{\text {hycell }}(r)$ computed by comparing the model fit with each of the 2292 radar-derived raincell profile $R_{c s r a d}(l)$.The average root mean square error (ARMSE, expressed in $\mathrm{mm} / \mathrm{h}$ ) between all radar measured $R_{c s}(l)$ and modeled $R_{c s \mathrm{MOD}}(l)$ (where MOD stands for EXP, GAU, and HYB) was also calculated as follows:

$\operatorname{ARMSE}=\frac{1}{N_{c s} N_{r}} \sum_{n=1}^{N_{c s}} \sum_{l=1}^{N_{r}}\left(R_{c s r a d}^{(n)}(l)-R_{c s \mathrm{MOD}}^{(n)}(l)\right)^{2}$

with $N_{c s}(=2292)$ is the number of considered single-peak raincells. As a further indicator of model fitting goodness, the correlation coefficient $\rho_{R}$ has been computed as follows:

$\rho_{R}=\frac{<\tilde{R}_{c s \mathrm{rad}}(l) \cdot \tilde{R}_{c s \mathrm{MOD}}(l)>}{\sqrt{<\tilde{R}_{c s \mathrm{rad}}^{2}(l)><\tilde{R}_{c s \mathrm{MOD}}^{2}(l)>}}$

where the angle brackets indicate the ensemble average as in Eq. (8) and the correlation coefficient has been defined by using variables centered (indicated by a tilde in the previous equation) with respect their average value. Comparing the results in terms of ARMSE and estimation correlation coefficient $\rho_{R}, R_{\mathrm{rad}}(r)$ it is clear that all raincell profile models are highly correlated even though the ARMSE may quite different with a better accuracy due to the HYB model. The ARMSE of the EXP model is better that that of the GAU model with a discrepancy of about $64 \%$, but the RMSE of the HYB is, in turn, much better than that of EXP of about $179 \%$.

To deepen the analysis of the rainrate profile modeling error, we have also analyzed the radial trend of RMSE along the radial distance $l$ from the raincell center, defined as:

$\operatorname{RMSE}(l)=\frac{1}{N_{c s}} \sum_{n=1}^{N_{c s}}\left(R_{c s r a d}^{(n)}(l)-R_{c s \mathrm{MOD}}^{(n)}(l)\right)^{2}$

The behavior of $R_{c s \mathrm{MOD}}^{(n)}(l)$ is illustrated in Fig. 5. The left panel shows that, up to about $6 \mathrm{~km}$, the error due to the EXP model is less than the GAU one, indicating that the latter shape is not always the worst form from this point of view. As a final plot, Fig. 6 shows the cumulative distribution function of the correlation coefficient $\rho_{R}$ between each radarobserved and best-fitted horizontal profile for the 3 considered models. The HYB model is again the most correlated and it has a better overall behavior with respect to other profile models, but at expenses of an increase of formulation complexity. 


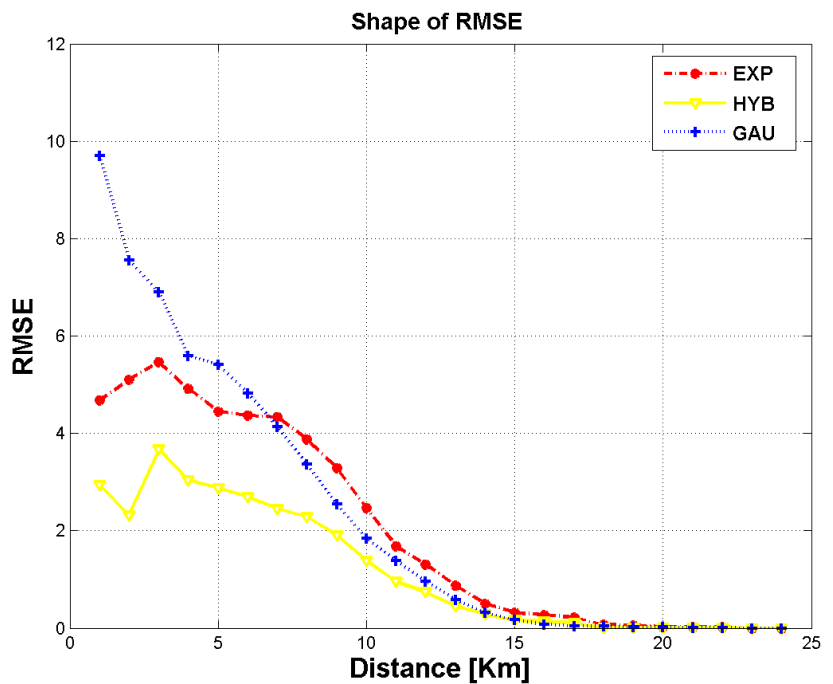

Fig. 5. Radial trend of the root means square error of the EXP, GAU and HYB models against the radial distance from the center of the single-peak raincell.

\section{Conclusions}

A spatial characterization of mid-latitude mesoscale rain fields from C-band radar measurements has been performed by means of a systematic analysis and modeling of convective raincell shapes. To this aim a large rainfall dataset, derived from an operational C-band dual-polarized radar and consisting of radar-derived rain intensities on a grid of $256 \times 256 \mathrm{~km}$ with a spatial resolution of $1 \mathrm{~km}$ from 1996 to 1999, has been collected. A new accurate algorithm for raincell identification has been introduced and discussed in detail. The major feature of the proposed recognition procedure is that it adopts variable and adapting thresholds, based on the spatial autocorrelation of the local rainfall field, in order to detect a raincell. From this analysis, a quality-controlled set of 2601 raincells has been extracted and a total number of 2292 single-peak horizontal profiles have been automatically identified.

Three one-dimensional analytical models of rainfall horizontal profile, such as the exponential (EXP), Gaussian (GAU) and hybrid (HYB), have been reviewed and tested by best fitting their parameters against estimated raincell data. The statistical results of this intercomparison have been quantitatively analyzed and discussed in terms of mean rainfall horizontal profiles and root mean square errors. Basing only on the concept of 1-D average profile and using the available C-band radar data, we have found out that the EXP model is more representative with respect to the Gaussian one at least up to $8 \mathrm{~km}$ from the center of the raincells. Analyzing all selected single-peak raincell profiles, it is confirmed the accurate behavior of the EXP model with respect to the GAU one, but the best agreement has been obtained using the HYB model. This means that 1-D raincell horizontal profile is well approximated by the combination of

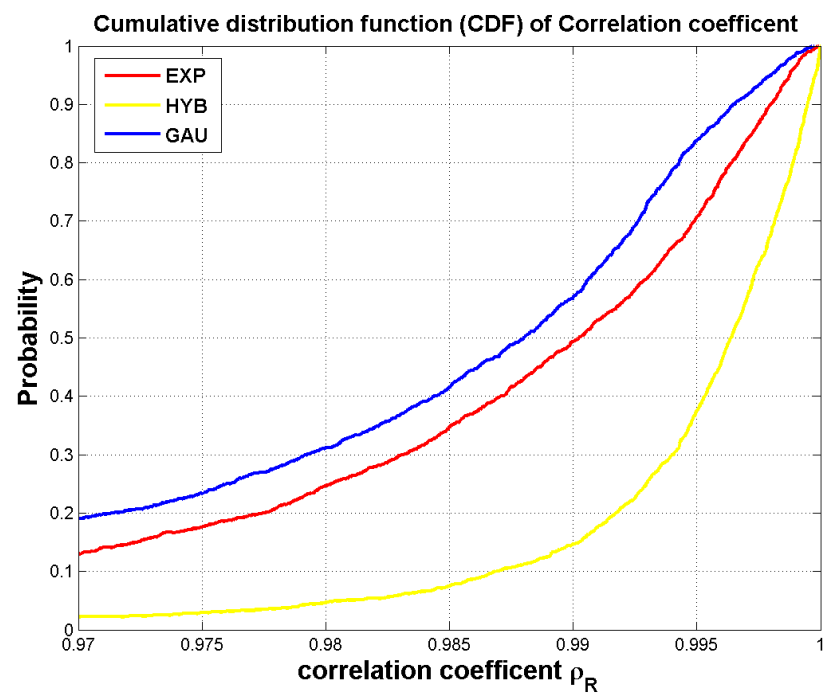

Fig. 6. The cumulative distribution function of the correlation coefficient $\rho_{R}$ for the 3 considered models.

a Gaussian function and an exponential one, that is by the model here called HYB. This result may be explained by considering that, from a conceptual point of view, the Gaussian component well describes the convective rainrate core of the raincell, whereas the exponential component accounts for the surrounding decaying or stratiform one. Of course, a better accuracy is opposed to a larger complexity of the hybrid-model pattern. A further improvement to be pursued in future works is to carry out this radar-based model intercomparison by introducing 2-D raincell analytical models.

\section{Appendix A Raincell horizontal profile models}

The definition of the analytical expressions of the one- dimensional (1-D) horizontal profile models, implemented in this work, is here briefly summarized. If $r$ is the radial distance from the raincell peak and $R(r)$ the radial rainrate profile, we will indicate $R_{\mathrm{EXP}}(r), R_{\mathrm{GAU}}(r), R_{\mathrm{HYB}}(r)$ the $1-\mathrm{D}$ model profiles defining the exponential (EXP), the Gaussian (GAU) and the hybrid (HYB) models as follows:

$$
\begin{aligned}
& R_{\mathrm{EXP}}(r)=R_{E} \cdot \exp \left(-\frac{r}{r_{E}}\right) \\
& R_{\mathrm{GAU}}(r)=R_{G} \cdot \exp \left\{-\frac{r^{2}}{r_{G}^{2}}\right\} \\
& R_{\mathrm{HYB}}(r)=\left\{\begin{array}{lll}
R_{\mathrm{HG}} \cdot \exp \left\{-\frac{r^{2}}{r_{\mathrm{HG}}^{2}}\right\} & \text { if } \quad R(r) \geq R_{\mathrm{thyb}} \\
R_{\mathrm{HE}} \cdot \exp \left\{-\frac{r}{r_{\mathrm{HE}}}\right\} & \text { if } \quad R(r)<R_{\mathrm{thyb}}
\end{array}\right.
\end{aligned}
$$

where $R_{\mathrm{E}}$ and $r_{\mathrm{E}}$ are the maximum and slope scale of EXP model, $R_{\mathrm{G}}$ and $r_{\mathrm{G}}^{2}$ are the maximum and variance scale of 
GAU model, and $\left(R_{\mathrm{HG}}, r_{\mathrm{HG}}^{2}\right)$ and $\left(R_{\mathrm{HE}}, r_{\mathrm{HE}}\right)$ are the maximum and scale of HYB model for the Gaussian and exponential component, respectively, with $R_{\text {thyb }}$ the rainrate separating threshold.

For the hybrid model, defined in (A3), a further continuity condition at the separation interface between the Gaussian and Exponential trend, is needed:

$R_{\mathrm{HG}} \cdot \exp \left\{-\frac{r_{t}^{2}}{r_{\mathrm{HG}}^{2}}\right\}=R_{\mathrm{thyb}}=R_{\mathrm{HE}} \cdot \exp \left\{-\frac{r_{t}}{r_{\mathrm{HE}}}\right\}$

where $r_{t}$ is the distance from the center of the raincell where the Gaussian and exponential portion of the hybrid model takes the same value equal at the separation threshold $R_{\text {thyb }}$.

The free parameters of the 1-D models, explained in Eqs. (A1), (A2), and (A3), are respectively:

1. the maximum $R_{\mathrm{E}}$ and the scale $r_{\mathrm{E}}$ where the profile $R_{\mathrm{EXP}}(r)$ decreases of $e^{-1}$;

2. the maximum $R_{\mathrm{G}}$ and the square scale $r_{\mathrm{G}}^{2}$ where the profile $R_{\mathrm{GAU}}(r)$ decrease of $\mathrm{e}^{-1}$;

3. the maximum $R_{\mathrm{HG}}$, the distances $r_{\mathrm{HG}}^{2}, r_{\mathrm{HE}}$, where the profile $R_{\mathrm{GAU}}(r)$ and $R_{\mathrm{EXP}}(r)$ decrease of $e^{-1}$ and the threshold separation $R_{\text {thyb }}$ between the Gaussian and exponential trend.

Thus, the free parameters of the HYB model are $R_{\mathrm{HG}}$, $r_{\mathrm{HG}}^{2}, r_{\mathrm{HE}}, R_{\mathrm{thyb}}$ which are computed by means of a minimization process between the observed and the modeled rainrate shape. The amplitude of the exponential portion $R_{\mathrm{HE}}$ of the hybrid model is not a free parameter because, from the continuity condition expressed by Eq. (A4), at the separation interface, $R_{\mathrm{HE}}$ is constrained to be dependent from the other parameters $R_{\mathrm{HG}}, r_{\mathrm{HG}}^{2}$ and $R_{\mathrm{thyb}}$.

Edited by: V. Kotroni and K. Lagouvardos

Reviewed by: anonymous referee

\section{References}

Alberoni, P. P., Andersson, T., Mezzasalma, P., Michelson, D. B, and Nanni, S.: Use of the vertical reflectivity profile for identification of anomalous propagation, Meteorol. Appl., 8, 257-266, 2001.

Austin, P. M. and Houze, R. A.: Analysis of structure of precipitation patterns in New England, J. Appl. Meteor., 11, 926-934, 1972.

Bell, T. L.: A space-time stochastic model of rainfall for satellite remote sensing studies, J. Geophys. Res., 92, D8, 9631-9643, 1987.

Capsoni, C., Fedi, F., and Paraboni, A.: A Comprehensively Meteorologically Oriented Methodology for the Prediction of Wave Propagation in Telecommunication Applications Beyond 10 GHz, Radio Sci., 22, 3, May-June 1987.
Crane, R. K.: Space-time structure of rain rate fields, J. Geophys. Res., 95, 2001-2020, 1990.

Féral, L., Mesnard, F., Sauvageot, H., Castanet, L., and Lemorton, J.: Rain Cell Shape and Orientation Distribution in South-West France, Phys. Chem. Earth B, 25, 1073-1078, 2000.

Féral, L., Sauvageot, H., Castanet, L., and Lemorton, J.: A new hybrid model of the rain horizontal distribution for propagation studies: 1. Modeling of the rain cell, Radio Sci., 38, 3, 10556, doi:10.1029/2002RS002802, 2003.

Ferraris, L., Gabellani, S., Rebora, N., and Provenzale, A.: A comparison of stochastic models for spatial rainfall downscaling", Water Resour. Res., 39, 12, 1368, doi:10.1029/2003WR002504, 2003.

Marzano, F. S., Turk, J., Ciotti, P., Di Michele, S., and Pierdicca, N.: Potential of combined spaceborne microwave and infrared radiometry for near real-time rainfall attenuation monitoring along earth-satellite links, Int. J. Satell. Commun., 19, 4, 385-412, 2001.

Marzano, F. S., Fionda, E., Ciotti, P., and Martellucci, A.: Groundbased multi-frequency microwave radiometry for rainfall remote sensing, IEEE Trans. Geosci. Rem. Sens., 40, 742-759, 2002.

Marzano, F. S., Picciotti, E., and Vulpiani, G.: Rain field and reflectivity vertical profile reconstruction from C-band radar volumetric data, IEEE Trans. Geosci. Remote Sens., 42, 4, 1033-1046, 2004.

Meija, J. and Rodriguez-Iturbe, I.: On the synthesis of random fields sampling from the spectrum: an application to the generation of hydrologic spatial processes, Water Resour. Res., 10, 705-711, 1974.

Mesnard, F. and Sauvageot, H.: Structural characteristics of rain fields, J. Geophys. Res., 108(D13), 4385, doi:10.1029/2002JD002808, 2003.

Pascual, R., Callado, A., and Berenguer, M.: Convective storm initiation in central Catalonia, Proc. of ERAD04, 464-468, 2004.

Pratt, W. K.: Digital image processing second edition, A Wileyinterscience publication, ISBN 0-471-85766-1, 1991.

Rigo, T. and Llasat, M. C.: A methodology for the classification of convective structures using methodology radar: Application to heavy rainfall events on the Mediterranean coast of the Iberian Peninsula, Nat. Hazards Earth Syst. Sci., 4, 59-68, 2004.

Sauvageot, H.: Radar Meteorology, Artech House, Norwood (MA), Boston-London, 1992.

Steiner, M., Houze, R. A., and Yuter, S. E.: Climatology Characterization of three-Dimensional Storm Structure from operational Radar and Rai Gauge Data, J. Applied Meteor., 34, 1978-2007, 1995.

Veneziano, D., Bras, R. L., and Niemann, J. D.: Nonlinearity and self-similarity of rainfall in time and a stochastic model, J. Geophys. Res., 101, D21, 26371-26392, 1996.

Von Hardenberg, J., Ferraris, L., and Provenzale, A.: The shape of convective rain cells, Geophys. Res. Lett., 30, 24, doi:10.1029/2003GL018 539, 2003.

Wheater, H. S., Isham, V. S., Cox, D. R., et al.: Spatial-temporal rainfall fields: modelling and statistical aspects, Hydrol. Earth Syst. Sci., 4, 581-601, 2000.

Willems, P.: A spatial rainfall generator for small spatial scales, J. Hydrol., 252, 126-144, 2001. 